\title{
Leader-Following Protocol Design for Switched Multiagent Systems with Randomly Occurring Self-Delay
}

\author{
M. J. Park, ${ }^{1}$ O. M. Kwon, ${ }^{1}$ Ju H. Park, ${ }^{2}$ S. M. Lee, ${ }^{3}$ and E. J. Cha ${ }^{4}$ \\ ${ }^{1}$ School of Electrical Engineering, Chungbuk National University, 52 Naesudong-ro, Heungdeok-gu, Cheongju 361-763, \\ Republic of Korea \\ ${ }^{2}$ Department of Electrical Engineering, Yeungnam University, 214-1 Dae-Dong, Gyeongsan 712-749, Republic of Korea \\ ${ }^{3}$ School of Electronic Engineering, Daegu University, Gyeongsan 712-714, Republic of Korea \\ ${ }^{4}$ Department of Biomedical Engineering, School of Medicine, Chungbuk National University, 52 Naesudong-ro, \\ Heungdeok-gu, Cheongju 361-763, Republic of Korea
}

Correspondence should be addressed to O. M. Kwon; madwind@chungbuk.ac.kr

Received 1 October 2013; Accepted 24 November 2013

Academic Editor: Shuping He

Copyright (C) 2013 M. J. Park et al. This is an open access article distributed under the Creative Commons Attribution License, which permits unrestricted use, distribution, and reproduction in any medium, provided the original work is properly cited.

\begin{abstract}
This paper designs a delay-dependent leader-following protocol for multiagent systems with both communication delay and randomly occurring self-delay. Based on the randomly occurring mode of self-delay, a new model of switched multi-agent systems which have not been introduced yet is constructed. By constructing a newly piecewise Lyapunov-Krasovskii functional, a leaderfollowing stability criterion of the switched multi-agent systems is derived by the framework of linear matrix inequalities (LMIs) and the average dwell time with the randomly occurring mode. Based on the result of the derived stability criterion, a designing leader-following protocol for the system will be proposed. One numerical example is included to show the effectiveness of the proposed method.
\end{abstract}

\section{Introduction}

During the last few years, Markovian jumping mode $[1,2]$, the randomly occurring mode with probabilistic property [3-5], the average dwell time approach [6-9], the indicator function definition with arbitrary mode [10], and other changing or switching signal modes are being put to treat in the various systems with changing or switching parameters. Among them, one pays close attention to the average dwell time approach with randomly occurring mode for the following two reasons.

(i) It is a well-known fact that when all linear systems in the family $\dot{x}(t)=A_{p} x(t), p \in \mathscr{P}=\{1,2, \ldots, m\}$ are stable, the switched linear system $\dot{x}(t)=A_{\sigma(t)} x(t)$, where $\sigma(t)$ is switching signal, is stable if the dwell time is sufficiently large [6]. Thus, a more general result of dwell time is the average dwell time.

(ii) Switching signals are generated based on the randomly occurring mode with probabilistic property.
Use of probabilistic property vouches for the simulation condition in corresponding to ever-changing practice situation.

On the other hand, multi-agent systems (MASs) [11] have received considerable attention due to their extensive applications in many fields such as distributed sensor networks [12], vehicle systems $[13,14]$, groups of mobile autonomous agent [15], multi-agent robotic system [16], and other applications [17-19]. Before handling this system, since modern systems use information between each agent in networks, these days, we need to pay keen attention to the three following considerations.

(i) A prime concern in this system is the agreement of a group of agent on their states of leader by interaction. Namely, this problem is a consensus problem with the leader.

(ii) During the information exchange between each agent in networks, there exists communication delay. Also, 
in implementation of many practical systems such as aircraft and electric circuits, there exist occasionally stochastic perturbations. The perturbations have influence on the random occurrence of self-delay.

(iii) It is well known that the time-delay often causes undesirable dynamic behaviors such as performance degradation and instability of various systems. Therefore, the study on stability analysis for systems with time-delay has been widely investigated [20-24]. Moreover, the analysis for systems with time-delay can be classified into two categories, namely, delaydependent analysis and delay-independent analysis. Here, delay-dependent analysis has been paid more attention than delay-independent one because the sufficient conditions for delay-dependent analysis make use of the information on the size of time delay [20]. That is, the former is less conservative than the latter particularly when the size of time-delay is small.

In this regard, the MASs are being put to use in the consensus problem for time-delay which occurs due to the finite speed of information processing and/or amplifier switching in the implementation of this system. Thus, it is necessary to study the problems for MASs with timedelay [25-28]. However, the above-mentioned literature has mainly addressed the consensus conditions of the MASs with only communication delay. In the point of practical systems, the randomly occurring self-delay is no less important than the communication delay as a considerable factor affecting dynamics in the fields of network science and communication systems applications. Unfortunately, to the best of authors' knowledge, the consensus problem for MASs with randomly occurring self-delay has not been tackled in any other literature, despite its potential and practical importance.

With this motivation mentioned above, in Section 2, based on the randomly occurring mode of self-delay, a new model of switched MASs is constructed for the first time. In Section 3, the problem to get a delay-dependent leaderfollowing protocol criterion for the switched MASs with both communication delay and randomly occurring selfdelay, which are interval time-varying delays, is considered. Here, delay-dependent stability or stabilization of system with interval time-varying delays has been a focused topic of theoretical and practical importance [29] in very recent years. The interval time-varying delays mean that the lower bounds of time-delay which guarantees the stability of system is not restricted to be zero. A networked control system is a typical example of dynamic systems with interval timevarying delays. So, the gist of the main points can be summarized as follows.

(i) It is assumed that the self-delay is of probabilistic property with Bernoulli sequence, which is more realistic in practical systems. For the details, see Assumption 2.

(ii) Switching signals are generated with not only the average dwell time, $\tau_{a}$, but also the occurrence probabilistic of self-delay, $\operatorname{Pr}\{\rho(t)=1\}$. Thus, in this paper, the new model of switched MASs is simulated with the probabilistic property with Bernoulli sequence. For the details, see the new model of switched MASs (11) in next section.

By construction of a newly piecewise LyapunovKrasovskii functional and utilization of some approaches, which are the average dwell time approach [6] and reciprocally convex approach [24] with some added decision variables, a new leader-following protocol criterion is derived in Theorem 12 with the LMI framework. The LMIs can be formulated as convex optimization algorithms which are amenable to computer solution [30]. Next, based on the results of Theorem 12, a leader-following protocol for the MASs with both communication delay and randomly occurring self-delay will be designed in Theorem 15. Finally, one numerical example is included to show the effectiveness of the proposed methods.

Notations. The notations used throughout this paper are fairly standard. $\mathbb{R}^{n}$ is the $n$-dimensional Euclidean space, and $\mathbb{R}^{m \times n}$ denotes the set of all $m \times n$ real matrices. For real symmetric matrices $X$ and $Y, X>Y$ (resp., $X \geq Y$ ) means that the matrix $X-Y$ is positive (resp., nonnegative) definite. $X^{\perp}$ denotes a basis for the null-space of $X . I_{n}, 0_{n}$, and $0_{m \cdot n}$ denote $n \times n$ identity matrix and $n \times n$ and $m \times n$ zero matrices, respectively. $\|\cdot\|$ refers to the Euclidean vector norm or the induced matrix norm. $\lambda_{\max }\{\cdot\}$ and $\lambda_{\min }\{\cdot\}$, respectively, denote the maximal and minimal eigenvalues of a given square matrix. $\operatorname{diag}\{\cdots\}$ denotes the block diagonal matrix. $\operatorname{Pr}\{A\}$ means the occurrence probability of the event $A . \otimes$ denotes the notation of Kronecker product.

\section{Problem Statements}

The interaction topology of a network of agents is represented using a directed graph (digraph) $\mathscr{G}=(\mathscr{V}, \mathscr{E}, \mathscr{A})$ with the set of nodes $\mathscr{V}=\{1,2, \ldots, N\}$ and edges $\mathscr{E}=\{(i, j): i, j \in \mathscr{V}\} \subset$ $\mathscr{V} \times \mathscr{V}$. An adjacency matrix $\mathscr{A}=\left[a_{i j}\right]_{N \times N}$ of the digraph $\mathscr{G}$ is the matrix with nonnegative elements satisfying $a_{i i}=0$ and $a_{i j} \geq 0$. If there is an edge between $i$ and $j$, then the elements of matrix $A$ are described as $a_{i j}>0 \Leftrightarrow(i, j) \in \mathscr{E}$. The digraph $\mathscr{G}$ is said to be undirected if $(i, j) \in \mathscr{E} \Leftrightarrow(j, i) \in \mathscr{E}$. A set of neighbors of agent $i$ is denoted by $\mathcal{N}_{i}=\{j \in \mathscr{V}:(i, j) \epsilon$ $\mathscr{E}$ \}. A degree of node $i$ is denoted by $\operatorname{deg}(i)=\sum_{j \in \mathcal{N}_{i}} a_{i j}$. A degree matrix of digraph $\mathscr{G}$ is diagonal and defined as $\mathscr{D}=$ $\operatorname{diag}\{\operatorname{deg}(1), \ldots, \operatorname{deg}(N)\}$. The Laplacian matrix $\mathscr{L}$ of graph $\mathscr{G}$ is defined as $\mathscr{L}=\mathscr{D}-\mathscr{A}$. More details can be seen in [31].

Consider the following MASs with the dynamics of agent $i$ :

$$
\dot{p}_{i}(t)=u_{i}(t), \quad \forall i \in \mathscr{V}
$$

where $N$ is the number of agents, $\mathscr{V}=\{1,2, \ldots, N\}$ is the index set, $n$ is the number of states of agent $i$, and $p_{i}(t) \epsilon$ $\mathbb{R}^{n}$ and $u_{i}(t) \in \mathbb{R}^{n}$ are the position vector and the leaderfollowing protocol vector of agent $i$, respectively. 


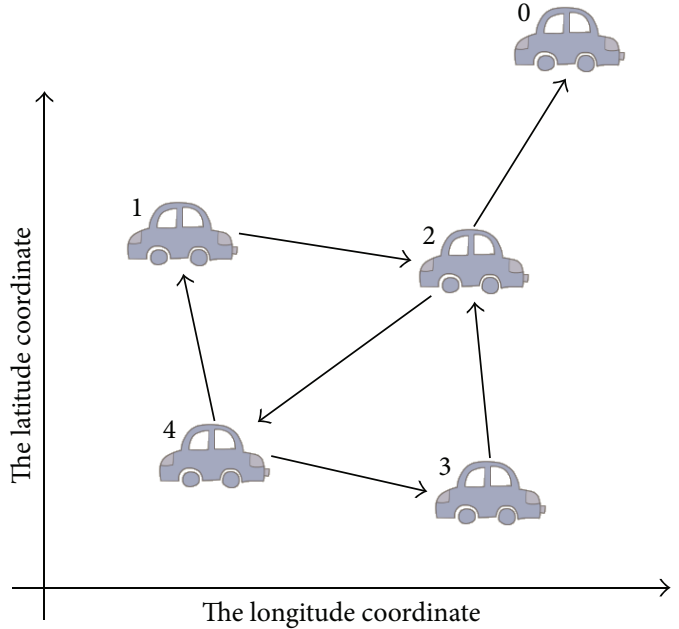

FIGURE 1: 4 vehicles in 2-dimensional plan.

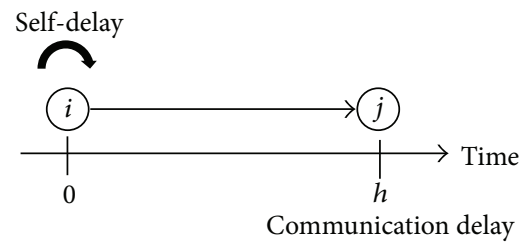

FIGURE 2: Concept for communication delay and self-delay.

According to the work [32], a leader-following algorithm in agent can be described as

$$
u_{i}(t)=-K \sum_{j \in \mathcal{N}_{i}} a_{i j}\left(p_{i}(t)-p_{j}(t)\right)-K b_{i}\left(p_{i}(t)-p_{0}\right)
$$

where $K \in \mathbb{R}^{n \times n}$ is a protocol gain matrix, $p_{0} \in \mathbb{R}^{n}$ is the position vector of leader, $a_{i j}$ and $b_{i}$ are the interconnection weights defining $a_{i j}>0$ if agent $i$ is connected to agent $j$, $a_{i j}=0$, otherwise, and $b_{i}>0$ if agent $i$ is connected to the leader, $b_{i}=0$, otherwise.

Remark 1. The agents of the MASs (1) with the leaderfollowing algorithm (2) can be known in the sense that each agent needs information from its local neighborhood. In practice, $N$-unmanned vehicles or $N$-soccer robots move in a $n$-dimensional plan with identical first-order dynamics. For example, the latitude and longitude coordinates of four vehicles shown in Figure 1, mean that $N=4$ and $n=2$.

Hence, the protocol gain $K$ is of the form of matrix, which is general or flexible, rather than the scalar.

Here, it is assumed that communication delay and selfdelay are as described in Figure 2.

Assumption 2. It is assumed that the self-delay is randomly occurring. This means that $\rho(t)$ is a probabilistic process representing the self-delay occurring process; that is, let $\rho(t)$ be a Bernoulli distributed sequence defined by

$$
\rho(t)= \begin{cases}1, & \text { if the self-delay occurs, } \\ 0, & \text { if the self-delay not occurs }\end{cases}
$$

where $\rho(t)$ satisfies $\operatorname{Pr}\{\rho(t)=1\}=\mathbb{E}\{\rho(t)\}=\rho_{0}, \operatorname{Pr}\{\rho(t)=$ $0\}=1-\rho_{0}$.

Remark 3. Commonly, in [33-35], the consensus problems with self-delay and without self-delay are addressed separately. However, we cannot regard that problem as something separate. Therefore, in this paper, the stochastic occurrence rate of self-delay is introduced in the consensus problem.

From algorithm (2) with Assumption 2, a leaderfollowing algorithm with probabilistic self-delay can be

$$
\begin{aligned}
u_{i}(t)= & -K_{\rho(t)} \sum_{j \in \mathcal{N}_{i}} a_{i j}(\underbrace{p_{i}(t-\rho(t) h(t))}_{\text {Term } 1}-\underbrace{p_{j}(t-h(t))}_{\text {Term } 2}) \\
& -K_{\rho(t)} b_{i}\left(p_{i}(t)-p_{0}\right),
\end{aligned}
$$

where $K_{\rho(t)}$ is a protocol gain matrix at $\rho(t)$, the terms 1 and 2 are, respectively, the self-delay and the communication delay, and the time-delay satisfies $0<h_{m} \leq h(t) \leq h_{M}$ and $\dot{h}(t) \leq$ $h_{D}$, where $h_{m}, h_{M}$, and $h_{D}$ are known constants.

Remark 4. It is common for the Bernoulli process to be changed to just random variable which belongs to the index set of two elements $\{0,1\}$, with $\operatorname{Pr}\{\rho(t)=1\}=\rho_{0}$. Hence, the Bernoulli process seems to be like an on-off switch; that is, it has two possible configurations. On the other hand, the selfdelay occurs by the calculational time delay or the processing limit time for the information in each agent. It depends on the real situation, and since the situation is random, the self-delay is explained with the Bernoulli process. Also, the proposed method in this work can also be extended to the MASs with randomly occurring communication delay given by

$$
\begin{aligned}
u_{i}(t)= & -K_{\rho(t)} \sum_{j \in \mathcal{N}_{i}} a_{i j}\left(p_{i}(t)-p_{j}(t-\rho(t) h(t))\right) \\
& -K_{\rho(t)} b_{i}\left(p_{i}(t)-p_{0}\right)
\end{aligned}
$$

or

$$
\begin{aligned}
u_{i}(t)= & -K_{\rho(t)} \sum_{j \in \mathcal{N}_{i}} a_{i j}\left(p_{i}(t-h(t))-p_{j}(t-\rho(t) h(t))\right) \\
& -K_{\rho(t)} b_{i}\left(p_{i}(t)-p_{0}\right) .
\end{aligned}
$$

This work will be carried out in our future research.

Remark 5. In practice, the self- and communication delay can have different or the same values. If we consider the systems with these delays of different value, then the analysis for the 
systems would be very complex. For the details, let us define each range of these delays as

$$
\begin{gathered}
d_{L}^{1} \leq d_{1}(t) \leq d_{U}^{1}, \quad \text { for self delay, } \\
d_{L}^{2} \leq d_{2}(t) \leq d_{U}^{2}, \quad \text { for communication delay. }
\end{gathered}
$$

Here, to allow for simple analysis, the time-delay $h(t)$ is set in the following range:

$$
\min _{i=1,2}\left\{d_{L}^{i}\right\} \leq h(t) \leq \max _{i i=1,2}\left\{d_{U}^{i}\right\} .
$$

Therefore, each interval of $d_{i}(t)$ belongs to the interval of $h(t)$ even if the intervals of $d_{i}(t)$ are different. Thus, in this paper, the analysis results for the concerned system with same timedelay $h(t)$ in self- and communication delay are guaranteed to the ones for the system with both these delays $d_{i}(t)$, like the analysis for convex-hull. Moreover, in this paper, $h_{m}=$ $\min _{i=1,2}\left\{d_{L}^{i}\right\}$ and $h_{M}=\max _{i=1,2}\left\{d_{U}^{i}\right\}$.

Remark 6. Such time-delays can coexist in the different terms of leader. It should be pointed out that the proposed method in this paper can also be extended to the network with timedelay between agents and leader. This work will be done for our future research topic.

Let us define $x_{i}(t)=p_{i}(t)-p_{0}$. From (1) and (4), the MASs with the error dynamics for agent $i$ can be rewritten as the matrix form by

$$
\begin{aligned}
\dot{x}(t)= & -\left(\mathscr{B} \otimes K_{\rho(t)}\right) x(t)+\left(\mathscr{A} \otimes K_{\rho(t)}\right) x(t-h(t)) \\
& -\left(\mathscr{D} \otimes K_{\rho(t)}\right) x(t-\rho(t) h(t)),
\end{aligned}
$$

where

$$
\begin{aligned}
& \mathscr{A}=\left[a_{i j}\right]_{N \times N}, \quad \mathscr{B}=\operatorname{diag}\left\{b_{1}, b_{2}, \ldots, b_{N}\right\}, \\
& \mathscr{D}=\operatorname{diag}\left\{\sum_{j \in \mathcal{N}_{1}} a_{1 j}, \sum_{j \in \mathcal{N}_{2}} a_{2 j}, \ldots, \sum_{j \in \mathcal{N}_{N}} a_{N j}\right\} .
\end{aligned}
$$

Remark 7. The notation $\rho(t)$ in system (9) is used to express the randomly occurring self-delay with Bernoulli distributed sequence. Instead of $\rho(t)$, a notation $\sigma(t)$ will be utilized as the switching signal when the system (9) is transformed into a new model of switched multi-agent systems. Moreover, compared with the existing works for the switched system, in this work, the switching rule between each mode is based on the probability property with Bernoulli distributed sequence.

For each $\rho(t)$, the system (9) can be rewritten as the following switched system:

$$
\dot{x}(t)=\left(F_{\sigma(t)} \otimes K_{\sigma(t)}\right) x(t)+\left(G_{\sigma(t)} \otimes K_{\sigma(t)}\right) x(t-h(t)),
$$

where $\mathscr{I}=\{1,2, \ldots, N\}$ is the index set and $\sigma(t)$ : $\mathbb{R} \rightarrow \mathscr{I}$ is the switching signal which is deterministic, piecewise constant, and right continuous. Correspondingly, the switching sequence $\left\{\left(i_{0}, t_{0}\right),\left(i_{1}, t_{1}\right), \ldots,\left(i_{l}, t_{l}\right), \ldots, \mid i_{l} \in\right.$ $\mathscr{I}, l=0,1, \ldots\}$ with $t_{0}=0$ means that the $i_{l}$ th subsystem is activated when $t \in\left[t_{l}, t_{l+1}\right)$, and, by understanding the considerations mentioned above,

$$
\begin{gathered}
F_{1}=-\mathscr{B}, \quad G_{1}=\mathscr{A}-\mathscr{D}, \quad \text { for } \sigma(t)=1(\rho(t)=1), \\
F_{2}=-(\mathscr{B}+\mathscr{D}), \quad G_{2}=\mathscr{A}, \quad \text { for } \sigma(t)=2(\rho(t)=0) .
\end{gathered}
$$

Also, the index set is defined as $\mathscr{I}=\{1,2\}$, and, for each switching signal $\sigma(t)$, we denote the matrices associated with mode $l \in \mathscr{I}$ by $F_{\sigma(t)}=F_{l}, G_{\sigma(t)}=G_{l}$, and $K_{\sigma(t)}=K_{l}$.

Remark 8. By representing the system (9) with probabilistic self-delay as the switching system (11), a deriving process of the criterion can be simple in analyzing the system (9) by utilizing the average dwell time approach [6]. Moreover, since the mathematical expectation operator $\mathbb{E}\{\cdot\}$ used in [35] is not needed, the deriving work for the leader-following criterion for MASs (1) with the leader-following algorithm (4) will be simple and easy. It should be noted that this approach has not been proposed yet.

The aim of this paper is to design the gains $K_{l}, \forall l \in \mathscr{I}$, which guarantees the leader-following protocol for the MASs with both communication delay and randomly occurring self-delay, in other words, the design of exponential stabilization controller gain $K_{l}$ for the switched multi-agent system (11). This means that the protocol $u_{i}(t)$ solves this leaderfollowing problem, if and only if the states of agents satisfy $\lim _{t \rightarrow \infty}\left\|x_{i}(t)\right\|=0, \forall i \in \mathscr{V}$. Therefore, the leader-following protocol gains $K_{l}$ will be obtained through a designing of the exponential stabilization controller gains $K_{l}$ for the switched multi-agent system (11). In order to do this, the following definitions and lemmas will be utilized.

Definition 9 (see [7-9]). System (11) is said to be exponentially stable under switching signal $\sigma(t)$ if the solution $x(t)$ of system (11) satisfies

$$
\|x(t)\| \leq \beta e^{-\gamma\left(t-t_{0}\right)}\left\|x\left(t_{0}\right)\right\|_{c 1}, \quad \forall t \geq t_{0},
$$

for constants $\beta \geq 1$ and $\gamma>0$, where $\|x(t)\|_{c 1}=$ $\sup _{-h_{M} \leq s \leq 0}\{\|x(t+s)\|,\|\dot{x}(t+s)\|\}$.

Definition 10 (see [6]). For any $t_{f}>t_{i} \geq 0$, let $N_{\sigma}(t, T)$ denote the switching number of $\sigma$ on an interval $\left(t_{i}, t_{f}\right)$, if $N_{\sigma}(t, T) \leq$ $N_{0}+(T-t) / \tau_{a}$ holds for given $N_{0} \geq 0$ and $\tau_{a}>0$. Then the constant $\tau_{a}$ is called the average dwell time and $N_{0}$ is the chatter bound. Without loss of generality, we choose $N_{0}=0$ in this paper.

Lemma 11 (Finsler's lemma; see [36]). Let $\zeta \in \mathbb{R}^{n}, \Phi=\Phi^{T} \in$ $\mathbb{R}^{n \times n}$, and $B \in \mathbb{R}^{m \times n}$ such that $\operatorname{rank}(B)<n$. The following statements are equivalent:

(i) $\zeta^{T} \Phi \zeta<0, \forall B \zeta=0, \zeta \neq 0$,

(ii) $B^{\perp^{T}} \Phi B^{\perp}<0$. 


\section{Main Results}

In this section, two theorems will be introduced. In Theorem 12, a leader-following stability criterion for system (11) will be introduced. Then, based on the result of Theorem 12, a leader-following protocol design for system (11) will be introduced in Theorem 15. Before introducing Theorem 12, some notations will be defined for simplicity of matrix representation. $\vartheta_{i} \in \mathbb{R}^{5 N n \times N n}(i=1,2, \ldots, 5)$ are defined as block entry matrices; for example, $\vartheta_{3}=$ $\left[0_{N n \cdot 2 N n}, I_{N n}, 0_{N n \cdot 2 N n}\right]^{T}$. The notations of several matrices are defined as

$$
\begin{aligned}
& \zeta^{T}(t) \\
& =\left[x^{T}(t), x^{T}\left(t-h_{m}\right), x^{T}(t-h(t)), x^{T}\left(t-h_{M}\right), \dot{x}^{T}(s)\right], \\
& \Upsilon_{l}=\left(F_{l} \otimes K_{l}\right) \vartheta_{1}^{T}+\left(G_{l} \otimes K_{l}\right) \vartheta_{3}^{T}-\vartheta_{5}^{T}, \\
& \Psi_{1 l}=\left[\begin{array}{c|c}
I_{N} \otimes R_{1 l} & -I_{N} \otimes R_{1 l} \\
\hline-I_{N} \otimes R_{1 l}^{T} & I_{N} \otimes R_{1 l}
\end{array}\right], \\
& \Psi_{2 l}=\left[\begin{array}{c|c}
I_{N} \otimes R_{2 l} & I_{N} \otimes W_{l} \\
\hline I_{N} \otimes W_{l}^{T} & I_{N} \otimes R_{2 l}
\end{array}\right], \\
& \mathcal{S}_{i l}=\left[\begin{array}{c|c}
I_{N} \otimes S_{i 1 l} & I_{N} \otimes S_{i 2 l} \\
\hline I_{N} \otimes S_{i 2 l}^{T} & I_{N} \otimes S_{i 3 l}
\end{array}\right] \quad(i=1,2), \\
& \mathscr{Z}_{i l}=\left[\begin{array}{c|c}
0_{N n} & I_{N} \otimes Z_{i l} \\
\hline I_{N} \otimes Z_{i l}^{T} & 0_{N n}
\end{array}\right] \quad(i=1,2,3), \\
& \Xi_{1 l}=\vartheta_{1}\left(I_{N} \otimes P_{l}\right) \vartheta_{5}^{T}+\vartheta_{5}\left(I_{N} \otimes P_{l}\right) \vartheta_{1}^{T}+\vartheta_{1}\left(I_{N} \otimes \alpha P_{l}\right) \vartheta_{1}^{T} \text {, } \\
& \Xi_{2 l}=\vartheta_{1}\left(I_{N} \otimes\left(Q_{1 l}+Q_{2 l}\right)\right) \vartheta_{1}^{T} \\
& -\vartheta_{2}\left(I_{N} \otimes e^{-\alpha h_{m}}\left(Q_{1 l}-Q_{3 l}\right)\right) \vartheta_{2}^{T} \\
& -\vartheta_{3}\left(I_{N} \otimes\left(1-h_{D}\right) e^{-\alpha h_{M}} Q_{2 l}\right) \vartheta_{3}^{T} \\
& -\vartheta_{4}\left(I_{N} \otimes e^{-\alpha h_{M}} Q_{3 l}\right) \vartheta_{4}^{T}, \\
& \Xi_{3 l}=\vartheta_{5}\left(I_{N} \otimes\left(h_{m}^{2} R_{1 l}+\left(h_{M}-h_{m}\right)^{2} R_{2 l}\right)\right) \vartheta_{5}^{T} \\
& -\left[\vartheta_{1}, \vartheta_{2}\right]\left(e^{-\alpha h_{m}} \Psi_{1 l}\right)\left[\vartheta_{1}, \vartheta_{2}\right]^{T} \\
& -\left[\vartheta_{2}-\vartheta_{3}, \vartheta_{3}-\vartheta_{4}\right]\left(e^{-\alpha h_{M}} \Psi_{2 l}\right) \\
& \times\left[\vartheta_{2}-\vartheta_{3}, \vartheta_{3}-\vartheta_{4}\right]^{T} \text {, } \\
& \Xi_{4 l}=\left[\vartheta_{1}, \vartheta_{5}\right]\left(h_{m} \mathcal{S}_{1 l}+\left(h_{M}-h_{m}\right) \mathcal{S}_{2 l}\right)\left[\vartheta_{1}, \vartheta_{5}\right]^{T},
\end{aligned}
$$

$$
\begin{gathered}
\Theta_{l}=\vartheta_{1}\left(I_{N} \otimes e^{-\alpha h_{m}} Z_{1 l}\right) \vartheta_{1}^{T} \\
-\vartheta_{2}\left(I_{N} \otimes\left(e^{-\alpha h_{m}} Z_{1 l}-e^{-\alpha h_{M}} Z_{2 l}\right)\right) \vartheta_{2}^{T} \\
-\vartheta_{3}\left(I_{N} \otimes e^{-\alpha h_{M}}\left(Z_{2 l}-Z_{3 l}\right)\right) \vartheta_{3}^{T} \\
-\vartheta_{4}\left(I_{N} \otimes e^{-\alpha h_{M}} Z_{3 l}\right) \vartheta_{4}^{T}, \\
\widetilde{\Xi}_{l}=\sum_{i=1}^{4} \Xi_{i l}+\Theta_{l},
\end{gathered}
$$

where the subscript $l$ means the switching signal $\sigma(t)$ at $l \epsilon$ $\mathscr{I}=\{1,2\}$; for example, $\widetilde{\Xi}_{1}=\widetilde{\Xi}_{\sigma(t)=1}$.

Now, the first result is given by Theorem 12 .

Theorem 12. For given scalars $0<h_{m} \leq h_{M}, h_{D}<1$, $\alpha>0$ and the gains $K_{1}, K_{2}$, the agents in the system (11) converge to the position of leader exponentially, if there exist positive definite matrices $P_{l} \in \mathbb{R}^{n \times n}, Q_{i l} \in \mathbb{R}^{n \times n}(i=1,2,3)$, $R_{i l} \in \mathbb{R}^{n \times n}(i=1,2)$, and $S_{i j l} \in \mathbb{R}^{n \times n}(i=1,2, j=1,3)$, any matrices $S_{i 2 l} \in \mathbb{R}^{n \times n}(i=1,2), W_{l} \in \mathbb{R}^{n \times n}$, and any symmetric matrices $Z_{i l} \in \mathbb{R}^{n \times n}(i=1,2,3)$ satisfying the following LMIs for $l=1,2$ :

$$
\begin{gathered}
{\left[\Upsilon_{l}^{\perp}\right]^{T} \widetilde{\Xi}_{l}\left[\Upsilon_{l}^{\perp}\right]<0,} \\
\Psi_{2 l} \geq 0, \\
\mathcal{S}_{1 l}+\mathscr{Z}_{1 l} \geq 0, \\
\mathcal{S}_{2 l}+\mathscr{Z}_{2 l} \geq 0, \\
\mathcal{S}_{2 l}+\mathscr{Z}_{3 l} \geq 0 .
\end{gathered}
$$

Then, system (11) is exponentially stable for any switching signal with the average dwell time satisfying

$$
\tau_{a}>\frac{\ln \mu}{\alpha}
$$

where $\mu \geq 1$ and satisfies the following for all $i, j \in \mathscr{I}$ :

$$
\begin{gathered}
P_{i} \leq \mu P_{j}, \quad Q_{p i} \leq \mu Q_{p j}, \quad R_{q i} \leq \mu R_{q j}, \\
{\left[\begin{array}{c|c}
S_{q 1 i} & S_{q 2 i} \\
\hline S_{q 2 i}^{T} & S_{q 3 i}
\end{array}\right] \leq \mu\left[\begin{array}{c|c}
S_{q 1 j} & S_{q 2 j} \\
\hline S_{q 2 j}^{T} & S_{q 3 j}
\end{array}\right] \quad(p=1,2,3, q=1,2) .}
\end{gathered}
$$

Moreover, an estimate of state decay is given by

$$
\|x(t)\| \leq \sqrt{\frac{b}{a}} e^{-\left(\left(\alpha-\ln \mu / \tau_{a}\right) / 2\right) t}\|x(0)\|_{c 1},
$$


where

$$
\begin{aligned}
& a=\min _{l=1,2} \lambda_{\min }\left\{P_{l}\right\}, \\
& b=\max _{l=1,2} \lambda_{\max }\left\{P_{l}\right\}+\max _{l=1,2} h_{m} \lambda_{\max }\left\{Q_{l l}\right\} \\
& +h_{M} \max _{l=1,2} \lambda_{\max }\left\{Q_{2 l}\right\}+\left(h_{M}-h_{m}\right) \\
& \times \max _{l=1,2} \lambda_{\max }\left\{Q_{3 l}\right\}+\frac{h_{m}^{3}}{2} \max _{l=1,2} \lambda_{\max }\left\{R_{1 l}\right\} \\
& +\frac{\left(h_{M}-h_{m}\right)^{3}}{2} \max _{l=1,2} \lambda_{\max }\left\{R_{2 l}\right\} \\
& +\frac{h_{m}^{2}}{2} \max _{l=1,2} \lambda_{\max }\left\{\left[\begin{array}{c|c}
S_{11 l} & S_{12 l} \\
\hline S_{12 l}^{T} & S_{13 l}
\end{array}\right]\right\} \\
& +\frac{\left(h_{M}-h_{m}\right)^{2}}{2} \max _{l=1,2} \lambda_{\max }\left\{\left[\begin{array}{l|l}
S_{21 l} & S_{22 l} \\
\hline S_{22 l}^{T} & S_{23 l}
\end{array}\right]\right\} .
\end{aligned}
$$

Proof. Let us consider the following Lyapunov-Krasovskii functional candidate as

$$
V_{\sigma(t)}(x(t))=V_{1 \sigma(t)}+V_{2 \sigma(t)}+V_{3 \sigma(t)}+V_{4 \sigma(t)},
$$

where

$$
\begin{gathered}
V_{1 \sigma(t)}=x(t)\left(I_{N} \otimes P_{\sigma(t)}\right) x(t), \\
V_{2 \sigma(t)}=\int_{t-h_{m}}^{t} x^{T}(s)\left(I_{N} \otimes e^{\alpha(s-t)} Q_{1 \sigma(t)}\right) x(s) d s \\
+\int_{t-h(t)}^{t} x^{T}(s)\left(I_{N} \otimes e^{\alpha(s-t)} Q_{2 \sigma(t)}\right) x(s) d s \\
+\int_{t-h_{M}}^{t-h_{m}} x^{T}(s)\left(I_{N} \otimes e^{\alpha(s-t)} Q_{3 \sigma(t)}\right) x(s) d s, \\
V_{3 \sigma(t)=} h_{m} \int_{t-h_{m}}^{t} \int_{s}^{t} \dot{x}^{T}(u)\left(I_{N} \otimes e^{\alpha(u-t)} R_{1 \sigma(t)}\right) \dot{x}(u) d u d s \\
+\left(h_{M}-h_{m}\right) \\
\times \int_{t-h_{M}}^{t-h_{m}} \int_{s}^{t} \dot{x}^{T}(u)\left(I_{N} \otimes e^{\alpha(u-t)} R_{2 \sigma(t)}\right) \dot{x}(u) d u d s, \\
V_{4 \sigma(t)}=\int_{t-h_{m}}^{t} \int_{s}^{t} \xi^{T}(u)\left(I_{N} \otimes e^{\alpha(u-t)} \mathcal{S}_{1 \sigma(t)}\right) \xi(u) d u d s \\
+\int_{t-h_{M}}^{t-h_{m}} \int_{s}^{t} \xi^{T}(u)\left(I_{N} \otimes e^{\alpha(u-t)} \mathcal{S}_{2 \sigma(t)}\right) \xi(u) d u d s,
\end{gathered}
$$

and $\xi^{T}(t)=\left[x^{T}(t), \dot{x}^{T}(t)\right]$.
The time-derivative of $V_{1 \sigma(t)}$ and $V_{2 \sigma(t)}$ can be calculated as

$$
\begin{aligned}
\dot{V}_{1 \sigma(t)}+\alpha V_{1 \sigma(t)}= & 2 x^{T}(t)\left(I_{N} \otimes P_{\sigma(t)}\right) \dot{x}(t)+\alpha V_{1 \sigma(t)} \\
= & \zeta^{T}(t) \Xi_{1 \sigma(t)} \zeta(t) \\
\dot{V}_{2 \sigma(t)}+\alpha V_{2 \sigma(t)} \leq & x^{T}(t)\left(I_{N} \otimes\left(Q_{1 \sigma(t)}+Q_{2 \sigma(t)}\right)\right) x(t) \\
& -x^{T}\left(t-h_{m}\right)\left(I_{N} \otimes e^{-\alpha h_{m}}\left(Q_{1 \sigma(t)}-Q_{3 \sigma(t)}\right)\right) \\
& \times x\left(t-h_{m}\right)-x^{T}(t-h(t)) \\
& \times\left(I_{N} \otimes\left(1-h_{D}\right) e^{-\alpha h_{M}} Q_{2 \sigma(t)}\right) x(t-h(t)) \\
& -x^{T}\left(t-h_{M}\right)\left(I_{N} \otimes e^{-\alpha h_{M}} Q_{3 \sigma(t)}\right) \\
& \times x\left(t-h_{M}\right) \\
= & \zeta^{T}(t) \Xi_{2 \sigma(t)} \zeta(t) .
\end{aligned}
$$

By use of Jensen's inequality [37], an upper bound of $\dot{V}_{3 \sigma(t)}$ is obtained as

$$
\begin{aligned}
& \dot{V}_{3 \sigma(t)}+\alpha V_{3 \sigma(t)} \\
& \leq \dot{x}^{T}(t)\left(I_{N} \otimes\left(h_{m}^{2} R_{1 \sigma(t)}+\left(h_{M}-h_{m}\right)^{2} R_{2 \sigma(t)}\right)\right) \dot{x}(t) \\
& -\int_{t-h_{m}}^{t} \dot{x}^{T}(s) d s\left(I_{N} \otimes e^{-\alpha h_{m}} R_{1 \sigma(t)}\right) \\
& \times \int_{t-h_{m}}^{t} \dot{x}(s) d s-\Phi(t), \\
& \text { where } \oplus(t)=\left(h_{M}-h(t)\right) /\left(h_{M}-h_{m}\right) \text { and } \\
& \Phi(t)=\frac{1}{1-\omega(t)} \int_{t-h(t)}^{t-h_{m}} \dot{x}^{T}(s) d s\left(I_{N} \otimes e^{-\alpha h_{M}} R_{2 \sigma(t)}\right) \\
& \times \int_{t-h(t)}^{t-h_{m}} \dot{x}(s) d s+\frac{1}{\omega(t)} \int_{t-h_{M}}^{t-h(t)} \dot{x}^{T}(s) d s \\
& \times\left(I_{N} \otimes e^{-\alpha h_{M}} R_{2 \sigma(t)}\right) \int_{t-h_{M}}^{t-h(t)} \dot{x}(s) d s .
\end{aligned}
$$

Here, when $h_{m}<h(t)<h_{M}$, since $\omega(t)$ satisfies $0<\omega(t)<1$, by reciprocally convex approach [24], the following inequality holds for any matrices $W_{\sigma(t)}$ :

$$
\begin{aligned}
0< & \frac{\emptyset(t)}{1-\varpi(t)} \int_{t-h(t)}^{t-h_{m}} \dot{x}^{T}(s) d s\left(I_{N} \otimes e^{-\alpha h_{M}} R_{2 \sigma(t)}\right) \\
& \times \int_{t-h(t)}^{t-h_{m}} \dot{x}(s) d s-\int_{t-h(t)}^{t-h_{m}} \dot{x}^{T}(s) d s \\
& \times\left(I_{N} \otimes e^{-\alpha h_{M}} W_{\sigma(t)}\right) \int_{t-h_{M}}^{t-h(t)} \dot{x}(s) d s \\
& -\int_{t-h_{M}}^{t-h(t)} \dot{x}^{T}(s) d s\left(I_{N} \otimes e^{-\alpha h_{M}} W_{\sigma(t)}^{T}\right) \\
& \times \int_{t-h(t)}^{t-h_{m}} \dot{x}(s) d s \frac{1-\varpi(t)}{\varpi(t)}
\end{aligned}
$$




$$
\begin{aligned}
& \times \int_{t-h_{M}}^{t-h(t)} \dot{x}^{T}(s) d s\left(I_{N} \otimes e^{-\alpha h_{M}} R_{2 \sigma(t)}\right) \\
& \times \int_{t-h_{M}}^{t-h(t)} \dot{x}(s) d s,
\end{aligned}
$$

which implies that

$$
\begin{aligned}
-\Phi(t)< & -\int_{t-h(t)}^{t-h_{m}} \dot{x}^{T}(s) d s\left(I_{N} \otimes e^{-\alpha h_{M}} R_{2 \sigma(t)}\right) \\
& \times \int_{t-h(t)}^{t-h_{m}} \dot{x}(s) d s \\
& -\int_{t-h(t)}^{t-h_{m}} \dot{x}^{T}(s) d s\left(I_{N} \otimes e^{-\alpha h_{M}} W_{\sigma(t)}\right) \\
& \times \int_{t-h_{M}}^{t-h(t)} \dot{x}(s) d s-\int_{t-h_{M}}^{t-h(t)} \dot{x}^{T}(s) d s \\
& \times\left(I_{N} \otimes e^{-\alpha h_{M}} W_{\sigma(t)}^{T}\right) \\
& \times \int_{t-h(t)}^{t-h_{m}} \dot{x}(s) d s-\int_{t-h_{M}}^{t-h(t)} \dot{x}^{T}(s) d s \\
& \times\left(I_{N} \otimes e^{-\alpha h_{M}} R_{2 \sigma(t)}\right) \\
& \times \int_{t-h_{M}}^{t-h(t)} \dot{x}(s) d s .
\end{aligned}
$$

It should be noted that when $h(t)=h_{m}$ or $h(t)=h_{M}$, we get $\int_{t-h(t)}^{t-h_{m}} \dot{x}(s) d s=\int_{t-h_{m}}^{t-h_{m}} \dot{x}(s) d s=0_{N n \cdot 1}$ or $\int_{t-h_{M}}^{t-h(t)} \dot{x}(s) d s=$ $\int_{t-h_{M}}^{t-h_{M}} \dot{x}(s) d s=0_{N n \cdot 1}$, respectively. Thus, if inequality (16) holds, then, an upper bound of the $\dot{V}_{3 \sigma(t)}$ can be rebounded as

$$
\dot{V}_{3 \sigma(t)}+\alpha V_{3 \sigma(t)} \leq \zeta^{T}(t) \Xi_{3 \sigma(t)} \zeta(t)
$$

Next, $\dot{V}_{4 \sigma(t)}$ is obtained as

$$
\begin{aligned}
\dot{V}_{4 \sigma(t)} & +\alpha V_{4 \sigma(t)} \\
= & \xi^{T}(t)\left(h_{m} \mathcal{S}_{1 \sigma(t)}+\left(h_{M}-h_{m}\right) \mathcal{S}_{2 \sigma(t)}\right) \xi(t) \\
& -\int_{t-h_{m}}^{t} \xi^{T}(s)\left(e^{-\alpha h_{m}} \mathcal{S}_{1 \sigma(t)}\right) \xi(s) d s \\
& -\int_{t-h(t)}^{t-h_{m}} \xi^{T}(s)\left(e^{-\alpha h_{M}} \mathcal{S}_{2 \sigma(t)}\right) \xi(s) d s \\
& -\int_{t-h_{M}}^{t-h(t)} \xi^{T}(s)\left(e^{-\alpha h_{M}} \mathcal{S}_{2 \sigma(t)}\right) \xi(s) d s .
\end{aligned}
$$

Before estimating $\dot{V}_{4 \sigma(t)}$, inspired by the work of [38], the following zero equality with any symmetric matrices $Z_{1 \sigma(t)}$, $Z_{2 \sigma(t)}$, and $Z_{3 \sigma(t)}$ holds as a less conservative tool

$$
\begin{aligned}
& 0=x^{T}(t)\left(I_{N} \otimes e^{-\alpha h_{m}} Z_{1 \sigma(t)}\right) x(t) \\
& -x^{T}\left(t-h_{m}\right)\left(I_{N} \otimes e^{-\alpha h_{m}} Z_{1 \sigma(t)}\right) x\left(t-h_{m}\right) \\
& -2 \int_{t-h_{m}}^{t} x^{T}(s)\left(I_{N} \otimes e^{-\alpha h_{m}} Z_{1 \sigma(t)}\right) \dot{x}(s) d s \\
& +x^{T}\left(t-h_{m}\right)\left(I_{N} \otimes e^{-\alpha h_{M}} Z_{2 \sigma(t)}\right) x\left(t-h_{m}\right) \\
& -x^{T}(t-h(t))\left(I_{N} \otimes e^{-\alpha h_{M}} Z_{2 \sigma(t)}\right) x(t-h(t)) \\
& -2 \int_{t-h(t)}^{t-h_{m}} x^{T}(s)\left(I_{N} \otimes e^{-\alpha h_{M}} Z_{2 \sigma(t)}\right) \dot{x}(s) d s \\
& +x^{T}(t-h(t))\left(I_{N} \otimes e^{-\alpha h_{M}} Z_{3 \sigma(t)}\right) x(t-h(t)) \\
& -x^{T}\left(t-h_{M}\right)\left(I_{N} \otimes e^{-\alpha h_{M}} Z_{3 \sigma(t)}\right) x\left(t-h_{M}\right) \\
& -2 \int_{t-h_{M}}^{t-h(t)} x^{T}(s)\left(I_{N} \otimes e^{-\alpha h_{M}} Z_{3 \sigma(t)}\right) \dot{x}(s) d s \\
& =\zeta^{T}(t) \Theta_{\sigma(t)} \zeta(t)-\int_{t-h_{m}}^{t} \xi^{T}(s)\left(e^{-\alpha h_{m}} \mathscr{Z}_{1 \sigma(t)}\right) \xi(s) d s \\
& -\int_{t-h(t)}^{t-h_{m}} \xi^{T}(s)\left(e^{-\alpha h_{M} \mathscr{Z}_{2 \sigma(t)}}\right) \xi(s) d s \\
& -\int_{t-h_{M}}^{t-h(t)} \xi^{T}(s)\left(e^{-\alpha h_{M} \mathscr{Z}_{3 \sigma(t)}}\right) \xi(s) d s .
\end{aligned}
$$

By adding (34) to (33), $\dot{V}_{4 \sigma(t)}$ can be estimated as

$$
\begin{aligned}
\dot{V}_{4 \sigma(t)}+ & \alpha V_{4 \sigma(t)} \\
= & \zeta^{T}(t)\left(\Xi_{4 \sigma(t)}+\Theta_{\sigma(t)}\right) \zeta(t) \\
& -\int_{t-h_{m}}^{t} \xi^{T}(s)\left(e^{-\alpha h_{m}}\left(\mathcal{S}_{1 \sigma(t)}+\mathscr{Z}_{1 \sigma(t)}\right)\right) \xi(s) d s \\
& -\int_{t-h(t)}^{t-h_{m}} \xi^{T}(s)\left(e^{-\alpha h_{M}}\left(\mathcal{S}_{2 \sigma(t)}+\mathscr{Z}_{2 \sigma(t)}\right)\right) \xi(s) d s \\
& -\int_{t-h_{M}}^{t-h(t)} \xi^{T}(s)\left(e^{-\alpha h_{M}}\left(\mathcal{S}_{2 \sigma(t)}+\mathscr{Z}_{3 \sigma(t)}\right)\right) \xi(s) d s .
\end{aligned}
$$

Thus, if inequalities (17)-(19) hold, then, an upper bound of $\dot{V}_{4 \sigma(t)}$ can be bounded as

$$
\dot{V}_{4 \sigma(t)}+\alpha V_{4 \sigma(t)} \leq \zeta^{T}(t)\left(\Xi_{4 \sigma(t)}+\Theta_{\sigma(t)}\right) \zeta(t) .
$$

Also, the system (11) with the augmented vector $\zeta(t)$ can be rewritten as

$$
\Upsilon_{\sigma(t)} \zeta(t)=0_{N n \cdot 1} .
$$


Then, from (26)-(36), a leader-following stability condition for the system (11) is

$$
\zeta^{T}(t) \widetilde{\Xi}_{\sigma(t)} \zeta(t)<0
$$

subject to (37). If inequality (38) holds, then

$$
\dot{V}_{\sigma(t)}(x(t))+\alpha V_{\sigma(t)}(x(t))<0 .
$$

From (37), the vector $\zeta(t)$ can be parameterized as

$$
\zeta(t)=\Upsilon_{\sigma(t)}^{\perp} y(t),
$$

where $r=\operatorname{rank}\left(\Upsilon_{\sigma(t)}\right)<N n$ and $y(t) \in \mathbb{R}^{r}$.

By utilizing Lemma 11 (ii) with (40), condition (38) is equivalent to the following inequality:

$$
\begin{aligned}
\zeta^{T}(t) \widetilde{\Xi}_{\sigma(t)} \zeta(t) & =\left(\Upsilon_{\sigma(t)}^{\perp} y(t)\right)^{T} \widetilde{\Xi}_{\sigma(t)}\left(\Upsilon_{\sigma(t)}^{\perp} y(t)\right) \\
& =y^{T}(t)\left\{\left[\Upsilon_{\sigma(t)}^{\perp}\right]^{T} \widetilde{\Xi}_{\sigma(t)}\left[\Upsilon_{\sigma(t)}^{\perp}\right]\right\} y(t)<0 .
\end{aligned}
$$

From inequality (41), if the LMIs (15)-(19) hold, then the presented leader-following stability condition (38) is satisfied.

Furthermore, according to the works [7-9], for an arbitrary piecewise constant switching signal $\sigma(t)$ and any $t>0$, assume that $t_{l}\left(l=1,2, \ldots, 0<t_{1}<t_{2}<\cdots\right)$ are the switching points of $\sigma(t)$ over the interval $(0, t)$. As mentioned above, the $i_{l}$ th subsystem is activated when $t \in\left[t_{l}, t_{l+1}\right)$. Integrating (39) from $t_{l}$ to $t$ gives

$$
V_{\sigma(t)}(x(t)) \leq e^{-\alpha\left(t-t_{l}\right)} V_{\sigma\left(t_{l}\right)}\left(x\left(t_{l}\right)\right) .
$$

Using (21) and (24), at the switching instant $t_{l}$, we get

$$
V_{\sigma\left(t_{l}\right)}\left(x\left(t_{l}\right)\right) \leq \mu V_{\sigma\left(t_{l}^{-}\right)}\left(x\left(t_{l}^{-}\right)\right) .
$$

Therefore, from (42), (43), and the relation $\kappa=N_{\sigma}(0, t) \leq$ $(t-0) / \tau_{a}$, it follows that

$$
\begin{aligned}
V_{\sigma(t)}( & x(t)) \\
\leq & e^{-\alpha\left(t-t_{l}\right)} \mu V_{\sigma\left(t_{l}^{-}\right)}\left(x\left(t_{l}^{-}\right)\right) \leq \cdots \leq e^{-\alpha(t-0)} \mu^{\kappa} \\
& \times V_{\sigma(0)}(x(0)) \\
\leq & e^{-\left(\alpha-\ln \mu / \tau_{a}\right) t} V_{\sigma(0)}(x(0)) .
\end{aligned}
$$

Furthermore, from (24), the following inequalities hold:

$$
\begin{gathered}
a\|x(t)\|^{2} \leq V_{\sigma(t)}(x(t)), \\
V_{\sigma(0)}(x(0)) \leq b\|x(0)\|_{c 1}^{2},
\end{gathered}
$$

where $a$ and $b$ are defined in (22). Combining (44) and (45) leads to

$$
a\|x(t)\|^{2} \leq V_{\sigma(t)}(x(t)) \leq b e^{-\left(\alpha-\ln \mu / \tau_{a}\right) t}\|x(0)\|_{c 1}^{2},
$$

which implies (22). Thus, by Definition 9 with $t_{0}=0$, the system (11) is exponentially stable. This completes our proof.
Remark 13. Since the proposed method is satisfied with condition (21), we have

$$
\alpha-\frac{\ln \mu}{\tau_{a}}>0,
$$

which implies that switching signal can guarantee that the switched system is stable; in other words, condition (47) corresponds to the exponential stability condition $\gamma>0$ in Definition 9.

Remark 14. When $\mu=1$ and $\alpha>0$, we have $\tau_{a}>$ $\ln \mu / \alpha=0$, which means that the switching signal can be arbitrary. At this time, with $\alpha=0$, we get the asymptotically stability condition for the concerned system. If $\mu>1$ and $\alpha \rightarrow 0$; that is, $\tau_{a} \rightarrow \infty$, then there is no switching. Also, the following condition means that a modeindependent Lyapunov-Krasovskii functional is required for all subsystems:

$$
\begin{aligned}
& P_{i}=P_{j}=P, \quad Q_{p i}=Q_{p j}=Q_{p}, \quad R_{q i}=R_{q j}=R_{q}, \\
& {\left[\begin{array}{c|l}
S_{q 1 i} & S_{q 2 i} \\
\hline S_{q 2 i}^{T} & S_{q 3 i}
\end{array}\right]=\left[\begin{array}{c|c}
S_{q 1 j} & S_{q 2 j} \\
\hline S_{q 2 j}^{T} & S_{q 3 j}
\end{array}\right]=\left[\begin{array}{c|l}
S_{q 1} & S_{q 2} \\
\hline S_{q 2}^{T} & S_{q 3}
\end{array}\right]} \\
& (p=1,2,3, q=1,2) \text {. }
\end{aligned}
$$

Moreover, in this paper, switching signals are generated with the occurrence probability of self-delay, $\operatorname{Pr}\{\rho(t)=1\}=\rho_{0}=$ $\operatorname{Pr}\{\sigma(t)=1\}$.

Theorem 12 provides the leader-following criterion for system (11) in the framework of LMIs when the leaderfollowing protocol gain is known. Based on the results of Theorem 12, we will propose a leader-following protocol design method for system (11) which will be introduced as follows.

Theorem 15. For given scalars $0<h_{m} \leq h_{M}, h_{D}<1$, and $\alpha>0$, the agents in the system (11) converge to the position of leader exponentially, if there exist positive definite matrices $P_{l} \in \mathbb{R}^{n \times n}, Q_{i l} \in \mathbb{R}^{n \times n}(i=1,2,3), R_{i l} \in \mathbb{R}^{n \times n}(i=1,2)$, and $S_{i j l} \in \mathbb{R}^{n \times n}(i=1,2, j=1,3)$, any matrices $S_{i 2 l} \in \mathbb{R}^{n \times n}(i=$ $1,2), W_{l} \in \mathbb{R}^{n \times n}$, and any symmetric matrices $Z_{i l} \in \mathbb{R}^{n \times n}(i=$ $1,2,3)$ satisfying LMIs (16)-(19) and

$$
\widetilde{\Xi}_{l}+\Gamma_{l}+\Gamma_{l}^{T}<0 \quad(l=1,2),
$$

where $\Gamma_{l}=\left[\vartheta_{1}+\vartheta_{5}\right]\left[\left(F_{l} \otimes Y_{l}\right) \vartheta_{1}^{T}+\left(G_{l} \otimes Y_{l}\right) \vartheta_{3}^{T}-\left(I_{N} \otimes X_{l}\right) \vartheta_{5}^{T}\right]$.

Then, the MASs (11) under the leader-following protocol gain $K_{l}=X_{l}^{-1} Y_{l}$ are exponentially stable.

Proof. Design the gains $K_{1}$ and $K_{2}$, with the same LyapunovKrasovskii functional candidate in (24), by using similar method in (26)-(36) and considering the following zero equalities with any matrices $X_{1}$ and $X_{2}$ :

$$
\begin{array}{r}
0=2 \zeta^{T}(t)\left[\vartheta_{1}\left(I_{N} \otimes X_{l}\right)+\vartheta_{5}\left(I_{N} \otimes X_{l}\right)\right] \Upsilon_{l} \zeta(t) \\
(l=1,2) .
\end{array}
$$




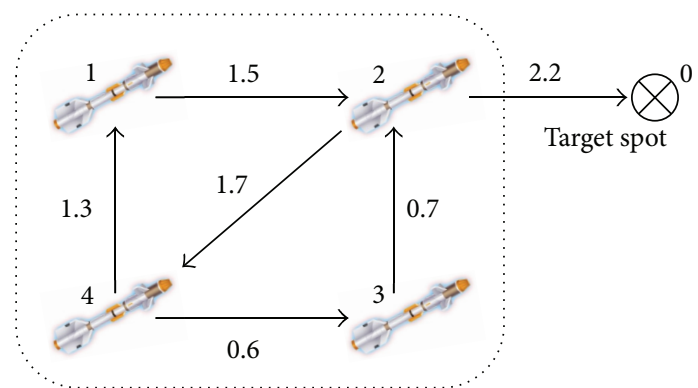

FIgURE 3: The interconnection topology.

Then, by defining $Y_{1}=X_{1} K_{1}$ and $Y_{2}=X_{2} K_{2}$, equalities (50) lead to

$$
\begin{aligned}
0=2 \zeta^{T}(t)\left[\vartheta_{1}+\vartheta_{5}\right][ & \left(F_{l} \otimes Y_{l}\right) \vartheta_{1}^{T}+\left(G_{l} \otimes Y_{l}\right) \vartheta_{3}^{T} \\
& \left.-\left(I_{N} \otimes X_{l}\right) \vartheta_{5}^{T}\right] \zeta(t) \quad(l=1,2) .
\end{aligned}
$$

Therefore, from (26)-(36), by consideration of zero equalities (51) and application of the S-procedure [30], a sufficient condition guaranteeing stability for the system (11) can be

$$
\zeta^{T}(t)\left(\sum_{i=1}^{4} \Xi_{i l}+\Theta_{l}+\Gamma_{l}+\Gamma_{l}^{T}\right) \zeta(t)<0 \quad(l=1,2) .
$$

In conclusion, condition (52) is equivalent to LMIs (49).

Remark 16. When information about the upper bound of $\dot{h}(t)$ is unknown, then by setting $Q_{2 \sigma(t)}=0$ in the Lyapunov-Krasovskii functional (24), the results with only the condition $0<h_{m} \leq h(t) \leq h_{M}$ can be obtained, and their proofs are similar to the proof of Theorems 12 and 15, so it is omitted.

\section{An Illustrative Example}

In this section, one numerical example will be shown to illustrate the effectiveness of the proposed Theorem 15. In an example, MATLAB LMI Control Toolbox [39] is used to solve LMI problems.

Consider MASs (11) with 4 missiles (agents) in 2dimensional plan (e.g., the latitude and longitude coordinate), that is, $N=4, n=2$, and the graph $\mathscr{G}$ of topology form described in Figure 3.

From Figure 1, the matrices $\mathscr{A}, \mathscr{B}$, and $\mathscr{D}$ related to graph $\mathscr{G}$ are represented as follow:

$$
\mathscr{A}=\left[\begin{array}{cccc}
0 & 1.5 & 0 & 0 \\
0 & 0 & 0 & 1.7 \\
0 & 0.7 & 0 & 0 \\
1.3 & 0 & 0.6 & 0
\end{array}\right]
$$

$$
\mathscr{B}=\operatorname{diag}\{1.5,1.7,0.7,1.9\}, \quad \mathscr{D}=\operatorname{diag}\{0,2.2,0,0\} \text {. }
$$

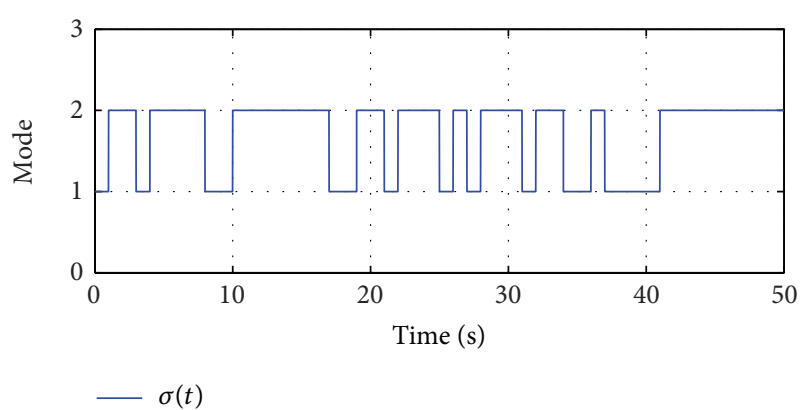

Figure 4: The curve of the operation modes.

Thus, in view of switched system (11), the parameters of each subsystem are

(i) subsystem 1 when $\sigma(t)=1($ for $\rho(t)=1)$

$$
F_{1}=-\mathscr{B}, \quad G_{1}=\mathscr{A}-\mathscr{D}=\left[\begin{array}{cccc}
-1.5 & 1.5 & 0 & 0 \\
0 & -1.7 & 0 & 1.7 \\
0 & 0.7 & -0.7 & 0 \\
1.3 & 0 & 0.6 & -1.9
\end{array}\right] \text {, }
$$

(ii) subsystem 2 when $\sigma(t)=2($ for $\rho(t)=0)$

$$
\begin{gathered}
F_{2}=-(\mathscr{B}+\mathscr{D})=-\operatorname{diag}\{1.5,3.9,0.7,1.9\}, \\
G_{2}=\mathscr{A} .
\end{gathered}
$$

For the system mentioned above, by applying Theorem 15, the leader-following protocol gains $K_{1}$ and $K_{2}$ and the range of average dwell time $\tau_{a}$ with fixed $\mu=1.05, \alpha=0.1, h_{D}=0.2$, and $h_{m}=0.1$ are

$$
\begin{aligned}
& K_{1}=\operatorname{diag}\{0.2074,0.2074\}, \\
& K_{2}=\operatorname{diag}\{0.2521,0.2521\},
\end{aligned}
$$

and $\tau_{a}>\ln \mu / \alpha=\ln 1.05 / 0.1=0.4879$. At this time, the maximum delay bound, $h_{M}$, is 0.76 . In order to confirm this result, we set the condition on time-delay as $h(t)=$ $0.1+0.66 \sin ^{2}(0.3 t)$. In addition, let us choose the occurrence probability of self-delay, $\rho_{0}$, as 0.7 . Then, the operation modes of switching signal are shown in Figure 4.

It is assumed that the position of leader is set by $p_{0}=$ $[5,-3]^{T}$. Then, the simulation results are given in Figure 5 . This figure shows that each agent with the responses converges to the position of leader under the protocol gains in (56) and the switching signal $\sigma(t)$.

Also, taking $\tau_{a}=1$, and from (22), we can further obtain the estimate of state decay

$$
\|x(t)\| \leq 16.2698 e^{-0.0256 t}\|x(0)\|_{c 1}=\chi(0),
$$

and its result is shown in Figure 6. 


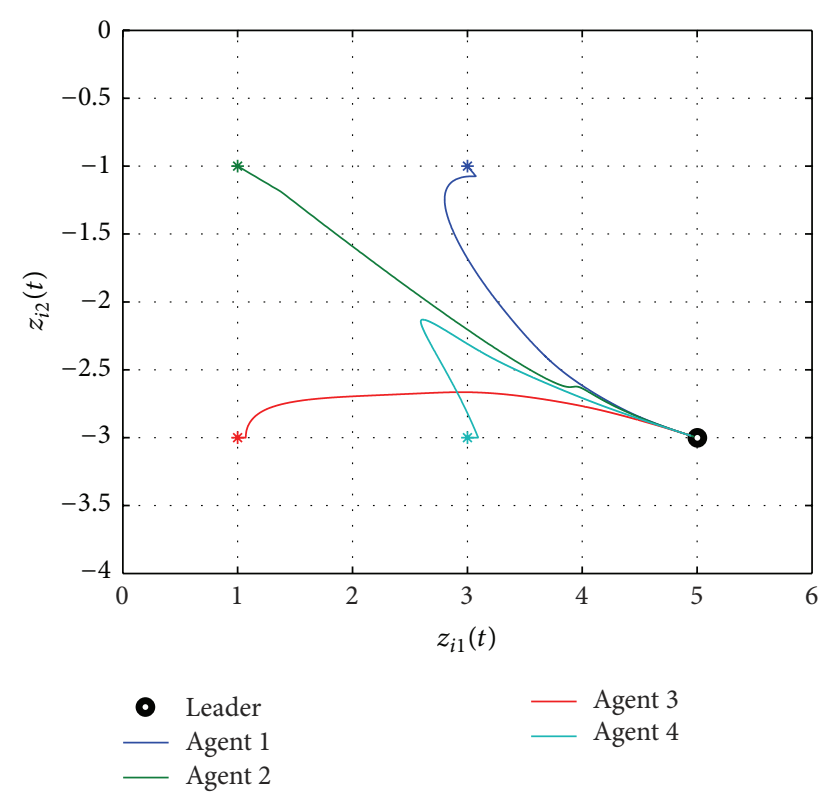

Figure 5: Positions of the agents and leader.

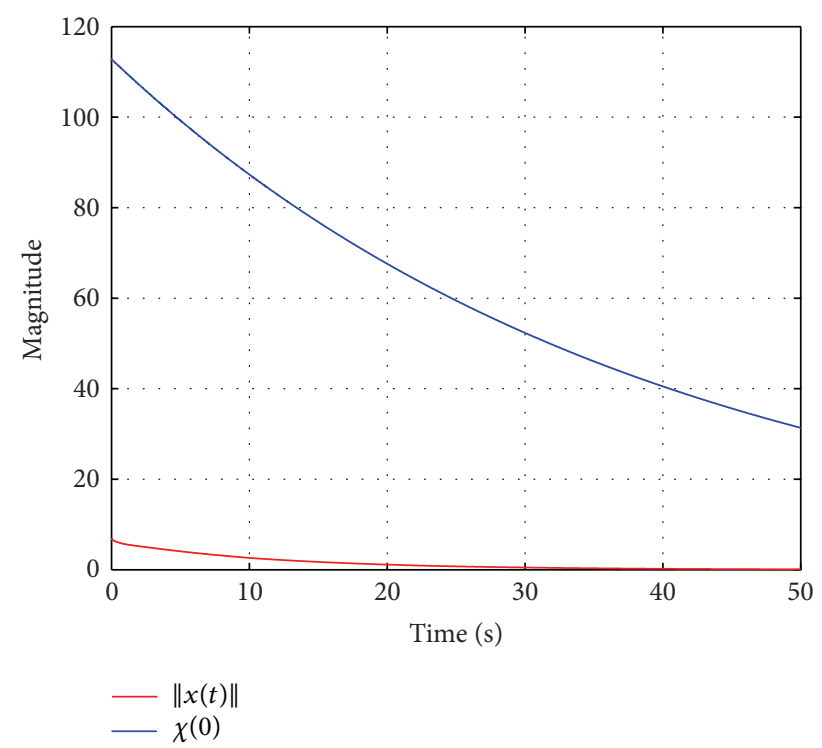

FIGURE 6: The estimate of state decay (57).

\section{Conclusions}

In this paper, the delay-dependent leader-following problem for the MASs with both communication delay and randomly occurring self-delay was studied. In Theorem 12, the leaderfollowing stability criterion for the MASs was proposed by introducing the new piecewise Lyapunov-Krasovskii functional and constructing the new model of switched MASs (11). In Theorem 15, based on the result of Theorem 12, the leader-following protocol gains for the switched MASs were designed. One illustrative example has been given to show the effectiveness and usefulness of the designed protocol gains.

\section{Acknowledgment}

This research was supported by the Basic Science Research Program through the National Research Foundation of Korea (NRF) funded by the Ministry of Education, Science and Technology (2011-0009273 and 2008-0062611).

\section{References}

[1] Z. G. Wu, P. Shi, H. Su, and J. Chu, "Passivity analysis for discrete-time stochastic markovian jump neural networks with mixed time delays," IEEE Transactions on Neural Networks, vol. 22, no. 10, pp. 1566-1575, 2011.

[2] M. J. Park, O. M. Kwon, J. H. Park, and S. M. Lee, "Simplified stability criteria for fuzzy Markovian jumping Hopfield neural networks of neutral type with interval time-varying delays," Expert Systems with Applications, vol. 39, no. 5, pp. 5625-5633, 2012.

[3] Z. Wang, Y. Wang, and Y. Liu, "Global synchronization for discrete-time stochastic complex networks with randomly occurred nonlinearities and mixed time delays," IEEE Transactions on Neural Networks, vol. 21, no. 1, pp. 11-25, 2010.

[4] Z. G. Wu, J. H. Park, H. Su, and J. Chu, "Robust dissipativity analysis of neural networks with time-varying delay and randomly occurring uncertainties," Nonlinear Dynamics, vol. 69, no. 3, pp. 1323-1332, 2012.

[5] J. Hu, Z. Wang, H. Gao, and L. K. Stergioulas, "Robust sliding mode control for discrete stochastic systems with mixed time delays, randomly occurring uncertainties, and randomly occurring nonlinearities," IEEE Transactions on Industrial Electronics, vol. 59, no. 7, pp. 3008-3015, 2012.

[6] D. Liberzon, Switching in Systems and Control, Systems \& Control: Foundations \& Applications, Birkhäuser, Boston, Mass, USA, 2003.

[7] Z. G. Wu, P. Shi, H. Su, and J. Chu, "Delay-dependent stability analysis for switched neural networks with time-varying delay," IEEE Transactions on Systems, Man, and Cybernetics B, vol. 41, no. 6, pp. 1522-1530, 2011.

[8] L. Wu and J. Lam, "Sliding mode control of switched hybrid systems with time-varying delay," International Journal of Adaptive Control and Signal Processing, vol. 22, no. 10, pp. 909-931, 2008.

[9] X. M. Sun, J. Zhao, and D. J. Hill, "Stability and $L_{2}$-gain analysis for switched delay systems: a delay-dependent method," Automatica, vol. 42, no. 10, pp. 1769-1774, 2006.

[10] C. K. Ahn, "An $H_{\infty}$ approach to stability analysis of switched Hopfield neural networks with time-delay," Nonlinear Dynamics, vol. 60, no. 4, pp. 703-711, 2010.

[11] R. Olfati-Saber, J. A. Fax, and R. M. Murray, "Consensus and cooperation in networked multi-agent systems," Proceedings of the IEEE, vol. 95, no. 1, pp. 215-233, 2007.

[12] W. Yu, G. Chen, Z. Wang, and W. Yang, "Distributed consensus filtering in sensor networks," IEEE Transactions on Systems, Man, and Cybernetics B, vol. 39, no. 6, pp. 1568-1577, 2009.

[13] W. Ren, "Consensus strategies for cooperative control of vehicle formations," IET Control Theory and Applications, vol. 1, no. 2, pp. 505-512, 2007.

[14] J. A. Fax and R. M. Murray, "Information flow and cooperative control of vehicle formations," IEEE Transactions on Automatic Control, vol. 49, no. 9, pp. 1465-1476, 2004.

[15] A. Jadbabaie, J. Lin, and A. S. Morse, "Coordination of groups of mobile autonomous agents using nearest neighbor rules," IEEE 
Transactions on Automatic Control, vol. 48, no. 6, pp. 988-1001, 2003.

[16] C. García, P. F. Cárdenas, L. J. Puglisi, and R. Saltaren, “Design and modeling of the multiagent robotic system: SMART," Robotics and Autonomous Systems, vol. 60, no. 2, pp. 143-153, 2012.

[17] P. DeLellis, M. diBernardo, F. Garofalo, and D. Liuzza, "Analysis and stability of consensus in networked control systems," Applied Mathematics and Computation, vol. 217, no. 3, pp. 9881000, 2010.

[18] D. Lee and M. W. Spong, "Stable flocking of multiple inertial agents on balanced graphs," IEEE Transactions on Automatic Control, vol. 52, no. 8, pp. 1469-1475, 2007.

[19] H. Kim, H. Shim, and J. H. Seo, "Output consensus of heterogeneous uncertain linear multi-agent systems," IEEE Transactions on Automatic Control, vol. 56, no. 1, pp. 200-206, 2011.

[20] S. Xu and J. Lam, "A survey of linear matrix inequality techniques in stability analysis of delay systems," International Journal of Systems Science, vol. 39, no. 12, pp. 1095-1113, 2008.

[21] O. M. Kwon, J. H. Park, and S. M. Lee, "On robust stability for uncertain neural networks with interval time-varying delays," IET Control Theory \& Applications, vol. 2, no. 7, pp. 625-634, 2008.

[22] O. M. Kwon, M. J. Park, J. H. Park, S. M. Lee, and E. J. Cha, "Improved robust stability criteria for uncertain discretetime systems with interval time-varying delays via new zero equalities," IET Control Theory \& Applications, vol. 6, no. 16, pp. 2567-2575, 2012.

[23] J. H. Kim, "Note on stability of linear systems with time-varying delay," Automatica, vol. 47, no. 9, pp. 2118-2121, 2011.

[24] P. G. Park, J. W. Ko, and C. Jeong, "Reciprocally convex approach to stability of systems with time-varying delays," Automatica, vol. 47, no. 1, pp. 235-238, 2011.

[25] P. A. Bliman and G. Ferrari-Trecate, "Average consensus problems in networks of agents with delayed communications," Automatica, vol. 44, no. 8, pp. 1985-1995, 2008.

[26] Y. P. Tian and C. L. Liu, "Consensus of multi-agent systems with diverse input and communication delays," IEEE Transactions on Automatic Control, vol. 53, no. 9, pp. 2122-2128, 2008.

[27] F. Xiao and L. Wang, "State consensus for multi-agent systems with switching topologies and time-varying delays," International Journal of Control, vol. 79, no. 10, pp. 1277-1284, 2006.

[28] J. Qin, H. Gao, and W. X. Zheng, "On average consensus in directed networks of agents with switching topology and time delay," International Journal of Systems Science, vol. 42, no. 12, pp. 1947-1956, 2011.

[29] V. L. Kharitonov and S. I. Niculescu, "On the stability of linear systems with uncertain delay," IEEE Transactions on Automatic Control, vol. 48, no. 1, pp. 127-132, 2003.

[30] S. Boyd, L. El Ghaoui, E. Feron, and V. Balakrishnan, Linear Matrix Inequalities in System and Control Theory, vol. 15 of SIAM Studies in Applied Mathematics, SIAM, Philadelphia, Pa, USA, 1994.

[31] C. Godsil and G. Royle, Algebraic Graph Theory, vol. 207 of Graduate Texts in Mathematics, Springer, New York, NY, USA, 2001.

[32] W. Ni and D. Cheng, "Leader-following consensus of multiagent systems under fixed and switching topologies," Systems \& Control Letters, vol. 59, no. 3-4, pp. 209-217, 2010.

[33] Z. Meng, W. Ren, Y. Cao, and Z. You, "Leaderless and leaderfollowing consensus with communication and input delays under a directed network topology," IEEE Transactions on Systems, Man, and Cybernetics B, vol. 41, no. 1, pp. 75-88, 2011.

[34] Y. P. Tian and C. L. Liu, "Robust consensus of multi-agent systems with diverse input delays and asymmetric interconnection perturbations," Automatica, vol. 45, no. 5, pp. 1347-1353, 2009.

[35] U. Münz, A. Papachristodoulou, and F. Allgöwer, "Delay robustness in consensus problems," Automatica, vol. 46, no. 8, pp. $1252-1265,2010$.

[36] M. C. de Oliveira and R. E. Skelton, "Stability tests for constrained linear systems," in Perspectives in Robust Control, vol. 268 of Lecture Notes in Control and Information Sciences, pp. 241-257, Springer, London, UK, 2001.

[37] K. Gu, "An integral inequality in the stability problem of time-delay systems," in Proceedings of the 39th IEEE Confernce on Decision and Control, pp. 2805-2810, Sydney, Australia, December 2000.

[38] S. H. Kim, P. Park, and C. K. Jeong, "Robust $H_{\infty}$ stabilisation of networked control systems with packet analyser," IET Control Theory and Applications, vol. 4, no. 9, pp. 1828-1837, 2010.

[39] P. Gahinet, A. Nemirovskii, A. Laub, and M. Chilali, LMI Control Toolbox, The MathWorks, Natick, Mass, USA, 1995. 


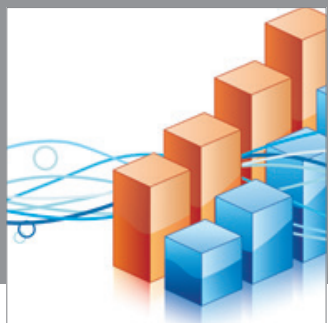

Advances in

Operations Research

mansans

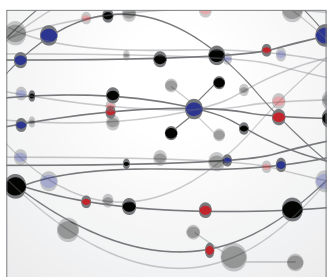

The Scientific World Journal
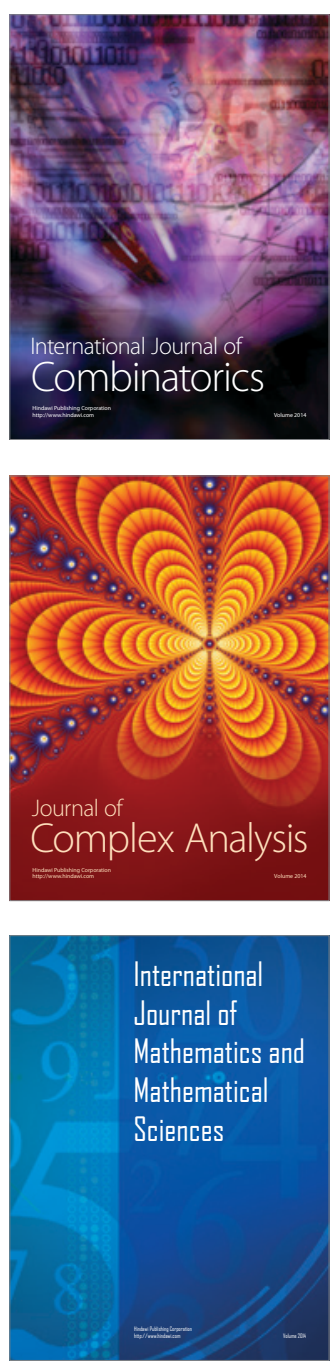
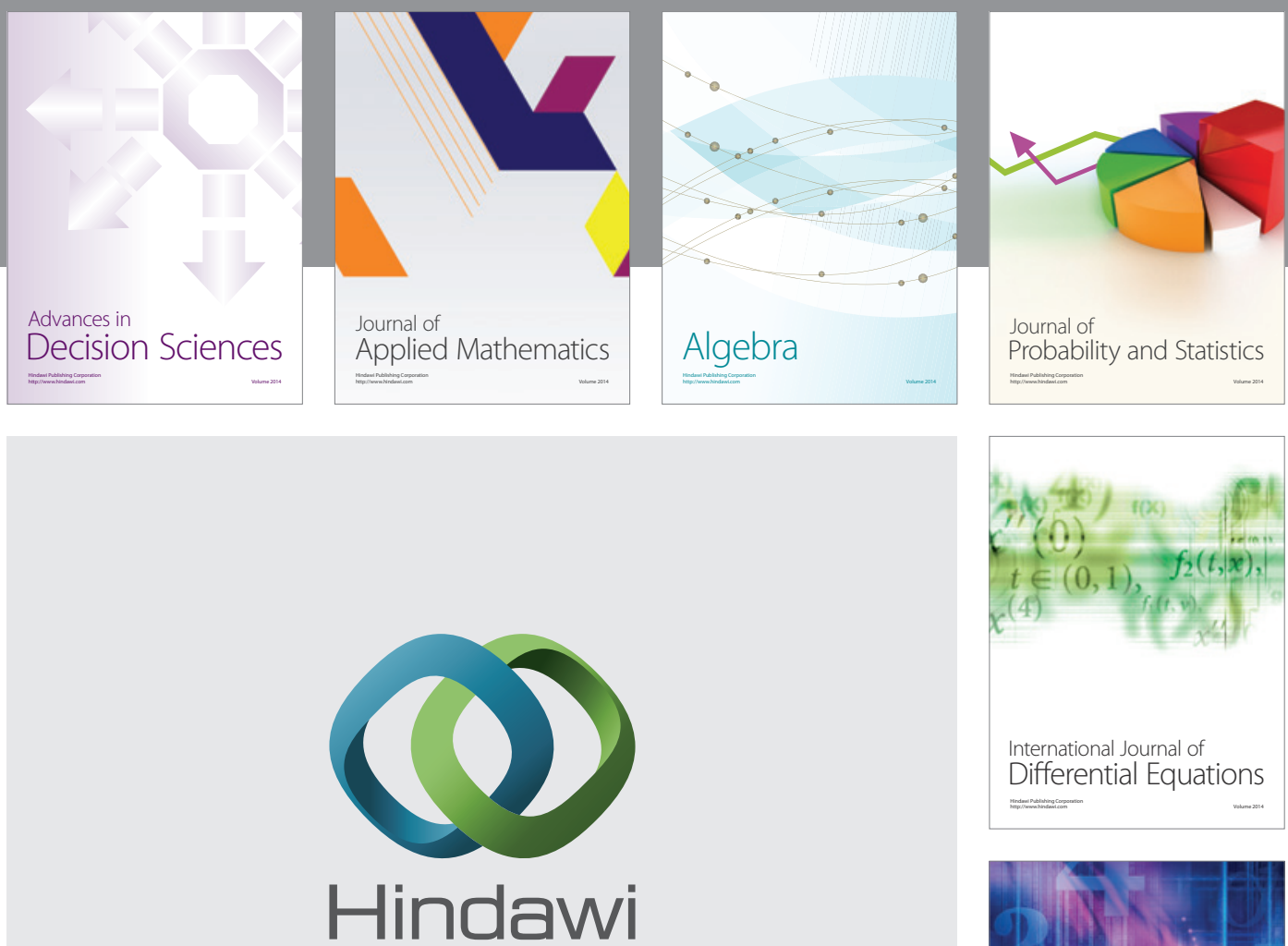

Submit your manuscripts at http://www.hindawi.com
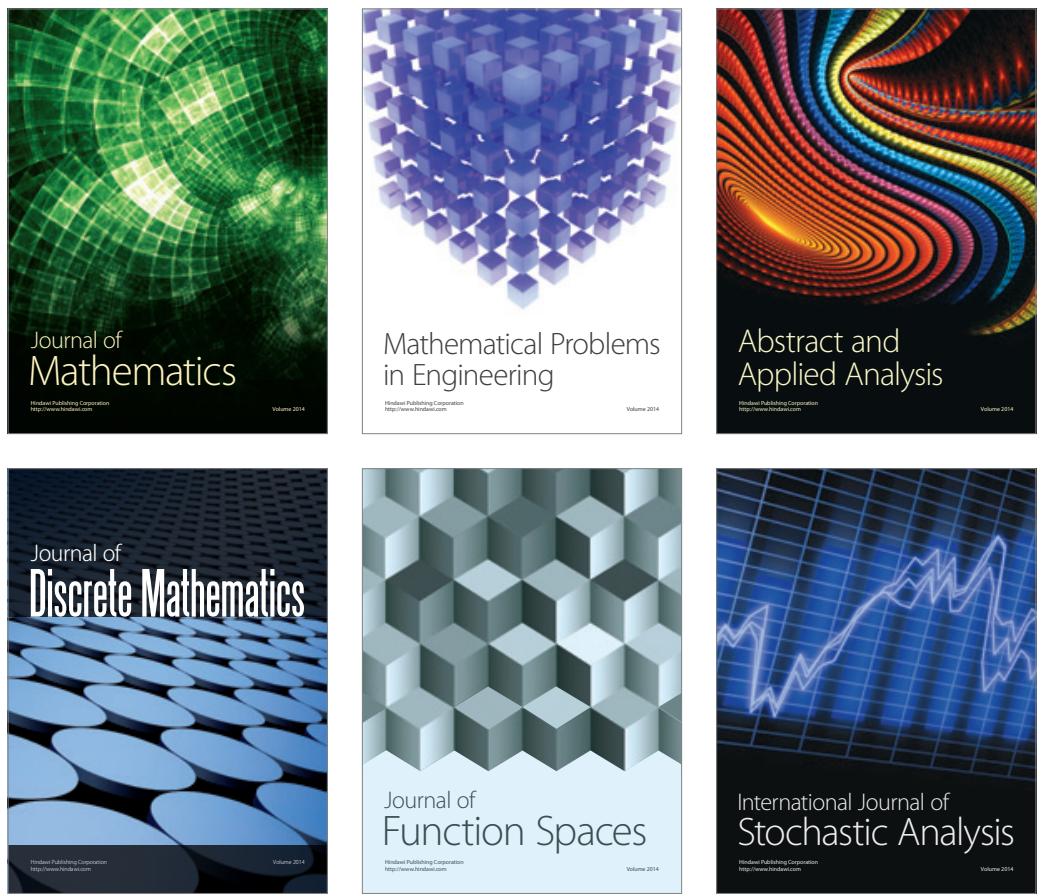

Journal of

Function Spaces

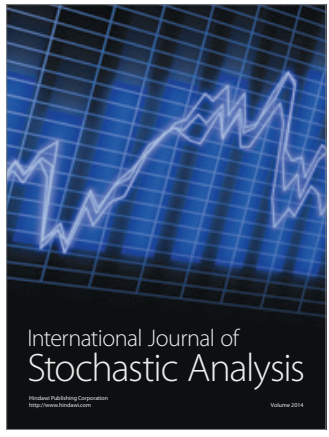

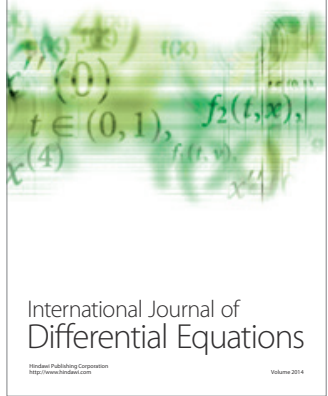
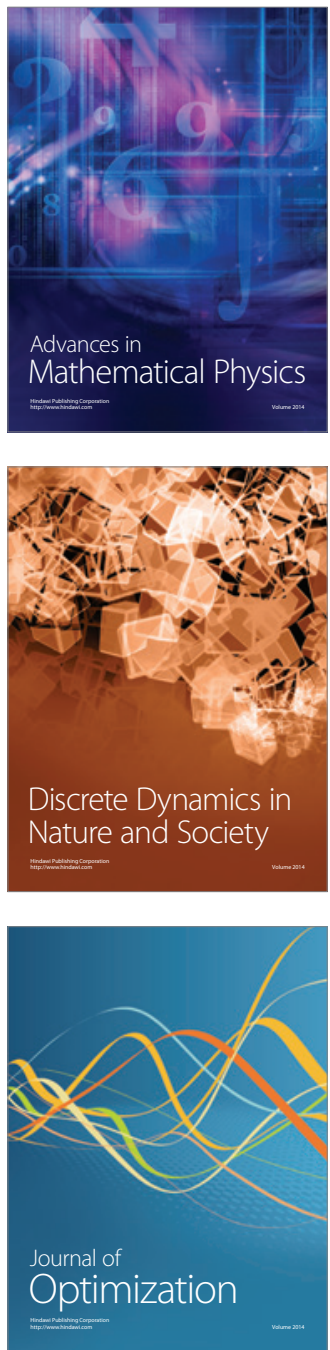